\title{
A semi-analytical method for bending, buckling and free vibration analyses of sandwich panels with square honeycomb cores
}

\author{
J. Liu
}

Department of Naval Architecture and Ocean Engineering, Huazhong University of Science and Technology, Wuhan 430074, P.R. China

\author{
F.T.K. $\mathrm{Au}^{*}$ \\ Department of Civil Engineering, The University of Hong Kong, Pokfulam Road, \\ Hong Kong, P.R. China
}

Y.S. Cheng

Department of Naval Architecture and Ocean Engineering, Huazhong University of Science and Technology, Wuhan 430074, P.R. China

R.F. Li

Department of Naval Architecture and Ocean Engineering, Huazhong University of Science and Technology, Wuhan 430074, P.R. China

Number of pages: 29

Number of tables: $\quad 4$

Number of figures: 7

* Corresponding author: $\quad$ Tel.: +852-2859 2650; fax: +852-2559 5337;

E-mail: francis.au@hku.hk 


\begin{abstract}
A semi-analytical method for bending, global buckling and free vibration analyses of sandwich panels with square honeycomb cores is presented. The discrete geometric nature of the square honeycomb core is taken into account by treating the core sheets as thin beams and the sandwich panel as composite structure of plates and beams with proper displacement compatibility. Based on the classical model of sandwich panels, the governing equations of motion of the discrete structure are derived using Hamilton's principle. Closed-form solutions are developed for bending, global buckling and free vibration of simply supported square-honeycomb sandwich panels by employing Fourier series and the Galerkin approach. Results from the proposed method agree well with available results in the literature and those from detailed finite element analysis. The effects of various geometric parameters of the sandwich panel on its behaviour are investigated. The present method provides an efficient way of analysis and optimization of sandwich panels with square honeycomb cores.
\end{abstract}

Keywords: bending, free vibration, global buckling, Hamilton's principle, sandwich panels with square honeycomb cores

\title{
1. Introduction
}

Sandwich panels have been widely used in marine, aviation and civil engineering. They have received much attention due to their light weight and higher stiffness to weight ratio than the solid plates of equal mass. A sandwich panel consists of two face sheets and a core. Commonly-used cores can be classified macroscopically into two groups, namely continuous (e.g. wood or metallic foam) and discrete (e.g. truss-core, honeycomb-core or corrugated-core of different geometry). The mechanical behaviour of sandwich panels has been extensively investigated in the past few decades. Many computational models for sandwich panels with continuous cores have been proposed. ${ }^{1,2}$

Compared with sandwich panels with continuous cores, the analysis of sandwich panels with discrete cores is more complicated due to the variation of geometrical forms of the cores. A lot of publications have been devoted to the analyses of sandwich panels with discrete cores. Libove and Hubka ${ }^{3}$ gave in 1951 the formulae for evaluating the equivalent elastic constants of sandwich plates with corrugated core. Sandwich panels with other types of core were also 
extensively investigated. ${ }^{4,5}$ Grediac $^{6}$ used the finite element method to obtain the transverse shear moduli of honeycomb sandwich panels and evaluated the effect of cell geometries. Subsequently to reduce the computations associated with the bending analysis of sandwich panels with Z-core, the equivalent elastic constants were derived by Fung et al. ${ }^{7}$ Recently Aimmanee and Vinson ${ }^{8}$ carried out the analysis and optimization of sandwich plates simply supported on all four edges with foam-reinforced web core subjected to in-plane compressive loads taking into account the overall instability of the sandwich, face wrinkling, and instability of the webs and face plates. Jayachandran et al. ${ }^{9}$ later investigated the buckling of sandwich plates by modelling sandwich plates as thin plates resting on elastic media. Romanoff and Varsta ${ }^{10,11}$ analyzed the bending response of web-core sandwich beams and plates respectively by transforming an originally discrete core into an equivalent homogenous continuum. Zok et al. ${ }^{12}$ and Rabczuk et al. ${ }^{13}$ carried out structural analysis of sandwich plates with pyramidal truss and tetrahedral truss cores, respectively. According to the finite element static analysis of sandwich panels with square honeycomb core performed by Kapania et al. ${ }^{14}$ in conjunction with the classical laminated plate theory (CLPT), the first-order shear deformation theory (FSDT) and the higher-order shear deformation theory (HSDT), as well as the equivalent single layer (ESL), the displacements predicted by the ESL finite element method are more accurate than those obtained by other methods. The homogenization theory has been employed by Buannic et al. ${ }^{15}$ to compute the effective properties of corrugated core sandwich panels. The shock resistance of sandwich plates with square honeycomb core has been extensively investigated. ${ }^{16,17}$ The experiments conducted by Cote et $a .^{18}$ to analyze the elastic and plastic buckling of metallic square-honeycombs have indicated that the metallic square-honeycombs out-perform some other cores. To study core behaviour, various constitutive models of the equivalent continuum of square honeycomb have been established through theoretical analysis and numerical simulation by Xue and Hutchinson, ${ }^{19}$ Xue et al. ${ }^{20}$ and Zok et al. ${ }^{21}$

As the discrete nature of cores complicates the analysis, various researchers ${ }^{22,23,24}$ often either replace the discrete core by an equivalent continuum or treat the whole sandwich plate as an equivalent orthotropic plate for approximate analysis. The static, buckling and free vibration responses of sandwich panels are highly sensitive to variations of some effective 
material properties, such as the transverse shear stiffness of the core. ${ }^{25}$ Errors in the calculation of properties of the equivalent continuum are unavoidable. For example, in the evaluation of in-plane elastic properties of honeycomb with standard beam theory, the interaction between the face plates and the core is not considered. ${ }^{26}$

A semi-analytical method for analyzing bending, global buckling and free vibration responses of square-honeycomb sandwich panels is proposed in this paper, in which the sandwich panel is regarded as a composite structure of plates and beams with the core sheets taken as thin beams to consider transverse flexural and shear deformations. Using the classical sandwich panel theory with displacement compatibility conditions, the governing equations of the sandwich panel with square honeycomb core are obtained by Hamilton's principle. The closed-form solutions for bending, global buckling and free vibration responses of simply supported square-honeycomb sandwich panels are derived by using Fourier series and the Galerkin approach. Validation of the proposed method is carried out by comparing the present results with available solutions in the literature and those obtained from three-dimensional (3D) finite element analyses. The effects of various geometric parameters of the sandwich panel on its behaviour will also be investigated.

\section{Theoretical background}

Figure 1 shows a typical square-honeycomb sandwich panel with the key dimensions, namely the face sheet thickness $t_{f}$, the core sheet thickness $t_{c}$, the core height $h_{c}$, the total panel height $h\left(h=h_{c}+2 t_{f}\right)$, and the core sheet spacing $L_{c}$. The present analysis is based on the linear elastic small deformation theory. In particular, Kirchhoff thin plate theory is used for the face sheets. The core is assumed to be incompressible ${ }^{2}$ in the thickness direction. The core sheets are treated as thin beams, which are considered in terms of transverse flexural and shear deformations, with the torsional deformation ignored.

\subsection{Displacement model}

The computational model of sandwich panels adopted is based on compatibility and the assumption that the planes of the core section and the faces remain plane after deformation 
but not with the same slope. $^{2}$ The transverse shear strains in the face sheets are neglected while that of the core is included on the basis of first-order shear deformable theory. Thus the displacement field may be expressed as follows:

(a) Face sheets

$$
\begin{aligned}
& u_{k}\left(x, y, z_{k}, t\right)=u_{o k}(x, y, t)-z_{k} w_{, x}(x, y, t) \\
& v_{k}\left(x, y, z_{k}, t\right)=v_{o k}(x, y, t)-z_{k} w_{, y}(x, y, t) \\
& w_{k}\left(x, y, z_{k}, t\right)=w(x, y, t) \quad(k=t \text { or } b)
\end{aligned}
$$

(b) Entire core

$$
\begin{gathered}
u_{c}\left(x, y, z_{c}, t\right)=u_{o c}(x, y, t)+z_{c} \phi_{x c}(x, y, t) \\
v_{c}\left(x, y, z_{c}, t\right)=v_{o c}(x, y, t)+z_{c} \phi_{y c}(x, y, t) \\
w_{c}\left(x, y, z_{c}, t\right)=w(x, y, t)
\end{gathered}
$$

where the notation is elaborated with reference to Figure 2. The dummy subscript $k$ below may be one of $t$ and $b$ that stand for the top and bottom face sheets respectively (i.e. $k=t$ or $b$ ), and $z_{k}$ is the vertical coordinate of each face sheet measured downwards from its mid-plane. The displacements at $z=z_{k}$ of each face sheet consist of the in-plane displacements $u_{k}\left(x, y, z_{k}, t\right)$ and $v_{k}\left(x, y, z_{k}, t\right)$ along $x$ and $y$ directions respectively and the transverse displacement $w_{k}\left(x, y, z_{k}, t\right)$. In particular, the in-plane displacements of each face sheet can be expressed in terms of the mid-plane displacements $u_{o k}(x, y, t)$ and $v_{o k}(x, y, t)$ along $x$ and $y$ directions respectively. Similarly, the displacements at $z=z_{c}$ of the core consist of the in-plane displacements $u_{c}\left(x, y, z_{c}, t\right)$ and $v_{c}\left(x, y, z_{c}, t\right)$ along $x$ and $y$ directions respectively and the transverse displacement $w_{c}\left(x, y, z_{c}, t\right)$, where $z_{c}$ is the vertical coordinate of the core measured downwards from the mid-plane of the core. The displacements of the core are characterized by the mid-plane displacements $u_{o c}(x, y, t)$ and $v_{o c}(x, y, t)$ along $x$ and $y$ directions respectively, rotations of the normal $\phi_{x c}(x, y, t)$ and $\phi_{y c}(x, y, t)$ of $y z$ and $z x$-planes respectively and the transverse mid-plane displacement $w(x, y, t)$ that is also the vertical mid-plane displacement of the face sheets due to the incompressible core assumption. As usual, the variable $t$ denotes time. 
The in-plane displacements of the mid-plane of the face sheets, $u_{o k}(x, y, t)$ and $v_{o k}(x, y, t)$, are determined through the compatibility of deformation at face-core interfaces, namely

(a) Upper face-core interface

$$
\begin{aligned}
& u_{o t}(x, y, t)=u_{o c}(x, y, t)-\frac{h_{c}}{2} \phi_{x c}(x, y, t)+\frac{t_{t}}{2} w_{, x}(x, y, t) \\
& v_{o t}(x, y, t)=v_{o c}(x, y, t)-\frac{h_{c}}{2} \phi_{y c}(x, y, t)+\frac{t_{t}}{2} w_{, y}(x, y, t)
\end{aligned}
$$

(b) Lower face-core interface

$$
\begin{aligned}
& u_{o b}(x, y, t)=u_{o c}(x, y, t)+\frac{h_{c}}{2} \phi_{x c}(x, y, t)-\frac{t_{b}}{2} w_{, x}(x, y, t) \\
& v_{o b}(x, y, t)=v_{o c}(x, y, t)+\frac{h_{c}}{2} \phi_{y c}(x, y, t)-\frac{t_{b}}{2} w_{, y}(x, y, t)
\end{aligned}
$$

Since the core sheets are thin, they are treated as thin beams. The approximate displacement relationships between the discrete core sheets and the whole core can be written as:

(a) Core sheets spanning along $x$ - axis

$$
\begin{gathered}
u_{c i}\left(x, z_{c}, t\right)=u_{c}\left(x, y_{i}, z_{c}, t\right)=u_{o c}\left(x, y_{i}, t\right)+z_{c} \phi_{x c}\left(x, y_{i}, t\right) \\
v_{c i}\left(x, z_{c}, t\right)=v_{c}\left(x, y_{i}, z_{c}, t\right)=v_{o c}\left(x, y_{i}, t\right)+z_{c} \phi_{y c}\left(x, y_{i}, t\right) \\
w_{c i}(x, t)=w\left(x, y_{i}, t\right)
\end{gathered}
$$

(b) Core sheets spanning along $y$ - axis

$$
\begin{gathered}
u_{c j}\left(y, z_{c}, t\right)=u_{c}\left(x_{j}, y, z_{c}, t\right)=u_{o c}\left(x_{j}, y, t\right)+z_{c} \phi_{x c}\left(x_{j}, y, t\right) \\
v_{c j}\left(y, z_{c}, t\right)=v_{c}\left(x_{j}, y, z_{c}, t\right)=v_{o c}\left(x_{j}, y, t\right)+z_{c} \phi_{y c}\left(x_{j}, y, t\right) \\
w_{c j}(y, t)=w\left(x_{j}, y, t\right)
\end{gathered}
$$

where $u_{c i}\left(x, z_{c}, t\right), v_{c i}\left(x, z_{c}, t\right)$ and $w_{c i}(x, t)$ are the displacements along $x$-, $y$ - and $z$-axes respectively of the ith core sheet spanning along $x$-axis and $y_{i}$ is the $y$-coordinate of the core sheet. Similarly, $u_{c j}\left(y, z_{c}, t\right), v_{c j}\left(y, z_{c}, t\right)$ and $w_{c j}(y, t)$ denote the displacements along $x$-, $y$ - and $z$-axes respectively of the $j$ th core sheet spanning along $y$-axis and $x_{j}$ is the $x$-coordinate of the core sheet.

Substituting the displacement relations given by Equations (1) to (6) into the strain-displacement equations of the classical theory of elasticity, the following relations for the face sheets are obtained. 


$$
\begin{gathered}
\varepsilon_{x x}^{k}=\frac{\partial u_{k}}{\partial x}=\frac{\partial u_{o k}}{\partial x}-z_{k} \frac{\partial^{2} w}{\partial x^{2}} \\
\varepsilon_{y y}^{k}=\frac{\partial v_{k}}{\partial y}=\frac{\partial v_{o k}}{\partial y}-z_{k} \frac{\partial^{2} w}{\partial y^{2}} \\
\gamma_{x y}^{k}=\frac{\partial u_{k}}{\partial y}+\frac{\partial v_{k}}{\partial x}=\frac{\partial u_{o k}}{\partial y}+\frac{\partial v_{o k}}{\partial x}-2 z_{k} \frac{\partial^{2} w}{\partial x \partial y} \quad(k=t \text { or } b)
\end{gathered}
$$

where $\varepsilon_{x x}^{k}, \varepsilon_{y y}^{k}$ and $\gamma_{x y}^{k}(k=t$ or $b)$ are respectively the normal strains along $x$ and $y$ directions and the in-plane shear strain of the face sheets. In the present analysis, only the normal strains and transverse shear strains are taken into account for the core sheets. The normal strain $\varepsilon_{x x}^{x i}$ and shear strain $\gamma_{x z}^{x i}$ of the $i$ th core sheet along the $x$-axis can be expressed respectively as

$$
\begin{gathered}
\varepsilon_{x x}^{x i}=\frac{\partial u_{c i}\left(x, z_{c}, t\right)}{\partial x}=\frac{\partial u_{o c}\left(x, y_{i}, t\right)}{\partial x}+z_{c} \frac{\partial \phi_{x c}\left(x, y_{i}, t\right)}{\partial x} \\
\gamma_{x z}^{x i}=\frac{\partial u_{c i}\left(x, z_{c}, t\right)}{\partial z_{c}}+\frac{\partial w_{c i}(x, t)}{\partial x}=\phi_{x c}\left(x, y_{i}, t\right)+\frac{\partial w\left(x, y_{i}, t\right)}{\partial x}
\end{gathered}
$$

while the normal strain $\varepsilon_{y y}^{y j}$ and shear strain $\gamma_{y z}^{y j}$ of the $j$ th core sheet along the $y$-axis appear respectively as

$$
\begin{gathered}
\varepsilon_{y y}^{y j}=\frac{\partial v_{c j}\left(y, z_{c}, t\right)}{\partial y}=\frac{\partial v_{o c}\left(x_{j}, y, t\right)}{\partial y}+z_{c} \frac{\partial \phi_{y c}\left(x_{j}, y, t\right)}{\partial y} \\
\gamma_{y z}^{y j}=\frac{\partial v_{c j}\left(y, z_{c}, t\right)}{\partial z_{c}}+\frac{\partial w_{c j}(y, t)}{\partial y}=\phi_{y c}\left(x_{j}, y, t\right)+\frac{\partial w\left(x_{j}, y, t\right)}{\partial y}
\end{gathered}
$$

\subsection{Constitutive relationship}

The materials of face sheets and core sheets are assumed to be isotropic. The constitutive relations for the face sheets can be written as

$$
\begin{aligned}
& \sigma_{x x}^{k}=\frac{E}{1-\mu^{2}}\left(\varepsilon_{x x}^{k}+\mu \varepsilon_{y y}^{k}\right) \\
& \sigma_{y y}^{k}=\frac{E}{1-\mu^{2}}\left(\varepsilon_{y y}^{k}+\mu \varepsilon_{x x}^{k}\right)
\end{aligned}
$$




$$
\tau_{x y}^{k}=\frac{E}{2(1+\mu)} \gamma_{x y}^{k} \quad(k=t \text { or } b)
$$

where $\sigma_{x x}^{k}, \sigma_{y y}^{k}$ and $\tau_{x y}^{k}$ are respectively the normal stresses along $x$ and $y$ directions and the in-plane shear stress of the face sheets, and $E$ and $\mu$ are Young's modulus and Poisson's ratio respectively. Treating the core sheets as one-dimensional thin beams, the stresses can be worked out easily. The normal stress $\sigma_{x x}^{x i}$ and shear stress $\tau_{x z}^{x i}$ of the ith core sheet along the $x$-axis can be expressed respectively as

$$
\begin{gathered}
\sigma_{x x}^{x i}=E_{c} \varepsilon_{x x}^{x i} \\
\tau_{x z}^{x i}=\frac{E_{c}}{2\left(1+\mu_{c}\right)} \gamma_{x z}^{x i}
\end{gathered}
$$

while the normal stress $\sigma_{y y}^{y j}$ and shear stress $\tau_{y z}^{y j}$ of the $j$ th core sheet along the $y$-axis appear respectively as

$$
\begin{gathered}
\sigma_{y y}^{y j}=E_{c} \varepsilon_{y y}^{y j} \\
\tau_{y z}^{y j}=\frac{E_{c}}{2\left(1+\mu_{c}\right)} \gamma_{y z}^{y j}
\end{gathered}
$$

where $E_{c}$ and $\mu_{c}$ are Young's modulus and Poisson's ratio of the material for the core respectively.

\subsection{Hamilton’s principle}

Hamilton's principle is employed to obtain the governing equations of the sandwich panels, namely

$$
\delta \int_{t_{1}}^{t_{2}}[K-(U+W)] d t=0
$$

where $K$ is the kinetic energy, $U$ is the total strain energy due to deformations, $W$ is the potential energy of the external loads and $\delta$ is the variation symbol. The variation of total strain energy can be expressed in terms of the strains and stresses as

$$
\begin{aligned}
& \delta U=\int_{V_{t}}\left(\sigma_{x x}^{t} \delta \varepsilon_{x x}^{t}+\sigma_{y y}^{t} \delta \varepsilon_{y y}^{t}+\tau_{x y}^{t} \delta \gamma_{x y}^{t}\right) d v+\int_{V_{b}}\left(\sigma_{x x}^{b} \delta \varepsilon_{x x}^{b}+\sigma_{y y}^{b} \delta \varepsilon_{y y}^{b}+\tau_{x y}^{b} \delta \gamma_{x y}^{b}\right) d v+ \\
& \sum_{i=1}^{m} \int_{V_{x i}}\left(\sigma_{x x}^{x i} \delta \varepsilon_{x x}^{x i}+\tau_{x z}^{x i} \delta \gamma_{x z}^{x i}\right) d v+\sum_{j=1}^{n} \int_{V_{y j}}\left(\sigma_{y y}^{y j} \delta \varepsilon_{y y}^{y j}+\tau_{y z}^{y j} \delta \gamma_{y z}^{y j}\right) d v
\end{aligned}
$$


where the first two terms are the strain energy of the top and bottom face sheets respectively, the last two terms are the strain energy of the core sheets spanning in $x$ - and $y$-axes respectively, $m$ and $n$ are the numbers of the core sheets along $x$-and $y$-axes respectively, and $d v$ is the differential volume. The variation of the kinetic energy of the sandwich panel can be written as

$$
\begin{aligned}
& \delta K=\delta\left[\frac{1}{2} \rho \int_{V_{t}}\left(\dot{u}_{t}^{2}+\dot{v}_{t}^{2}+\dot{w}_{t}^{2}\right) d v+\frac{1}{2} \rho \int_{V_{b}}\left(\dot{u}_{b}^{2}+\dot{v}_{b}^{2}+\dot{w}_{b}^{2}\right) d v+\right. \\
& \left.\sum_{i=1}^{m} \frac{1}{2} \rho_{c} \int_{V_{x i}}\left(\dot{u}_{c i}^{2}+\dot{v}_{c i}^{2}+\dot{w}_{c i}^{2}\right) d v+\sum_{j=1}^{n} \frac{1}{2} \rho_{c} \int_{V_{y j}}\left(\dot{u}_{c j}^{2}+\dot{v}_{c j}^{2}+\dot{w}_{c j}^{2}\right) d v\right]
\end{aligned}
$$

where the first two terms denote the kinetic energy of the top and bottom face sheets respectively, the last two terms denote the kinetic energy of the core sheets spanning in $x$ - and $y$-axes respectively, $\rho$ and $\rho_{c}$ are the mass densities of materials of the face sheets and core sheets respectively, and the dot denotes differentiation with respect to time $t$.

The sandwich panel is acted upon by a transverse distributed load $P(x, y, t)$ and external in-plane forces, which result in mid-plane normal forces $N_{x}$ and $N_{y}$ in $x$-and $y$-directions respectively and in-plane shear force $N_{x y}$ in the xy-plane. The variation of potential energy of the external load $\delta W$ can be written in terms of the differential area $d s$ along each component sheet as

$$
\delta W=\delta \int_{S} P(x, y, t) w(x, y) d s+\frac{1}{2} \delta \int_{S}\left[N_{x}\left(\frac{\partial w}{\partial x}\right)^{2}+2 N_{x y} \frac{\partial w}{\partial x} \frac{\partial w}{\partial y}+N_{y}\left(\frac{\partial w}{\partial y}\right)^{2}\right] d s
$$

By substituting the corresponding equations into Equation (13), integrating the resulting expression by parts, and collecting the coefficients of $\delta u_{o c}, \delta v_{o c}, \delta \phi_{x c}, \delta \phi_{y c}$ and $\delta w$, the following equations of motion are obtained:

$$
\begin{gathered}
\frac{2 E t_{f}}{1-\mu^{2}} \frac{\partial^{2} u_{o c}}{\partial x^{2}}+\frac{E t_{f}}{1+\mu} \frac{\partial^{2} u_{o c}}{\partial y^{2}}+\frac{E t_{f}}{1-\mu} \frac{\partial^{2} v_{o c}}{\partial x \partial y}+\sum_{i=1}^{m} E_{c} h_{c} t_{c} \frac{\partial^{2} u_{o c}}{\partial x^{2}} \delta_{D}\left(y-y_{i}\right) \\
=2 \rho t_{f} \frac{\partial^{2} u_{o c}}{\partial t^{2}}+\sum_{i=1}^{m} \rho_{c} h_{c} t_{c} \frac{\partial^{2} u_{o c}}{\partial t^{2}} \delta_{D}\left(y-y_{i}\right)+\sum_{j=1}^{n} \rho_{c} h_{c} t_{c} \frac{\partial^{2} u_{o c}}{\partial t^{2}} \delta_{D}\left(x-x_{j}\right) \\
\frac{E t_{f}}{1+\mu} \frac{\partial^{2} v_{o c}}{\partial x^{2}}+\frac{2 E t_{f}}{1-\mu^{2}} \frac{\partial^{2} v_{o c}}{\partial y^{2}}+\frac{E t_{f}}{1-\mu} \frac{\partial^{2} u_{o c}}{\partial x \partial y}+\sum_{j=1}^{n} E_{c} h_{c} t_{c} \frac{\partial^{2} v_{o c}}{\partial y^{2}} \delta_{D}\left(x-x_{j}\right) \\
=2 \rho t_{f} \frac{\partial^{2} v_{o c}}{\partial t^{2}}+\sum_{j=1}^{n} \rho_{c} h_{c} t_{c} \frac{\partial^{2} v_{o c}}{\partial t^{2}} \delta_{D}\left(x-x_{j}\right)+\sum_{j=1}^{n} \rho_{c} h_{c} t_{c} \frac{\partial^{2} v_{o c}}{\partial t^{2}} \delta_{D}\left(x-x_{j}\right)
\end{gathered}
$$




$$
\begin{aligned}
& \frac{E t_{f} h_{c}^{2}}{2\left(-1+\mu^{2}\right)} \frac{\partial^{2} \phi_{x c}}{\partial x^{2}}+\frac{E t_{f}^{2} h_{c}}{2\left(1-\mu^{2}\right)} \frac{\partial^{3} w}{\partial x^{3}}+\frac{E t_{f} h_{c}^{2}}{4(\mu-1)} \frac{\partial^{2} \phi_{y c}}{\partial x \partial y}+\frac{E t_{f}^{2} h_{c}}{2\left(1-\mu^{2}\right)} \frac{\partial^{3} w}{\partial y^{2} \partial x}-\frac{E t_{f} h_{c}^{2}}{4(1+\mu)} \frac{\partial^{2} \phi_{x c}}{\partial y^{2}} \\
& +\sum_{i=1}^{m} \frac{E_{c} h_{c} t_{c}}{2\left(1+\mu_{c}\right)}\left(\phi_{x c}+\frac{\partial w}{\partial x}\right) \delta_{D}\left(y-y_{i}\right)-\sum_{i=1}^{m} \frac{E_{c} h_{c}^{3} t_{c}}{12} \frac{\partial^{2} \phi_{x c}}{\partial x^{2}} \delta_{D}\left(y-y_{i}\right)=-\frac{1}{2} \rho t_{f} h_{c}^{2} \frac{\partial^{2} \phi_{x c}}{\partial t^{2}} \\
& +\frac{1}{2} \rho t_{f}^{2} h_{c} \frac{\partial^{3} w}{\partial x \partial t^{2}}-\sum_{i=1}^{m} \rho_{c} \frac{h_{c}^{3} t_{c}}{12} \frac{\partial^{2} \phi_{x c}}{\partial t^{2}} \delta_{D}\left(y-y_{i}\right)-\sum_{j=1}^{n} \rho_{c} \frac{h_{c}^{3} t_{c}}{12} \frac{\partial^{2} \phi_{x c}}{\partial t^{2}} \delta_{D}\left(x-x_{j}\right) \\
& \frac{E t_{f} h_{c}^{2}}{4(\mu-1)} \frac{\partial^{2} \phi_{x c}}{\partial x \partial y}+\frac{E t_{f}^{2} h_{c}}{2\left(1-\mu^{2}\right)} \frac{\partial^{3} w}{\partial y \partial x^{2}}-\frac{E t_{f} h_{c}^{2}}{4(1+\mu)} \frac{\partial^{2} \phi_{y c}}{\partial x^{2}}+\frac{E t_{f} h_{c}^{2}}{2\left(-1+\mu^{2}\right)} \frac{\partial^{2} \phi_{y c}}{\partial y^{2}}+\frac{E t_{f}^{2} h_{c}}{2\left(1-\mu^{2}\right)} \frac{\partial^{3} w}{\partial y^{3}} \\
& +\sum_{j=1}^{n} \frac{E_{c} h_{c} t_{c}}{2\left(1+\mu_{c}\right)}\left(\phi_{y c}+\frac{\partial w}{\partial y}\right) \delta_{D}\left(x-x_{j}\right)-\sum_{j=1}^{n} \frac{E_{c} h_{c}^{3} t_{c}}{12} \frac{\partial^{2} \phi_{y c}}{\partial y^{2}} \delta_{D}\left(x-x_{j}\right)=-\frac{1}{2} \rho t_{f} h_{c}^{2} \frac{\partial^{2} \phi_{y c}}{\partial t^{2}} \\
& +\frac{1}{2} \rho t_{f}^{2} h_{c} \frac{\partial^{3} w}{\partial y \partial t^{2}}-\sum_{i=1}^{m} \rho_{c} \frac{h_{c}^{3} t_{c}}{12} \frac{\partial^{2} \phi_{y c}}{\partial t^{2}} \delta_{D}\left(y-y_{i}\right)-\sum_{j=1}^{n} \rho_{c} \frac{h_{c}^{3} t_{c}}{12} \frac{\partial^{2} \phi_{y c}}{\partial t^{2}} \delta_{D}\left(x-x_{j}\right) \\
& \frac{4 E t_{f}^{3}}{3\left(1-\mu^{2}\right)} \frac{\partial^{4} w}{\partial x^{2} \partial y^{2}}-\frac{E t_{f}^{2} h_{c}}{2\left(1-\mu^{2}\right)} \frac{\partial^{3} \phi_{y c}}{\partial y^{3}}+\frac{2 E t_{f}^{3}}{3\left(1-\mu^{2}\right)} \frac{\partial^{4} w}{\partial y^{4}}-\frac{E t_{f}^{2} h_{c}}{2\left(1-\mu^{2}\right)} \frac{\partial^{3} \phi_{x c}}{\partial y^{2} \partial x}-\frac{E t_{f}^{2} h_{c}}{2\left(1-\mu^{2}\right)} \frac{\partial^{3} \phi_{x c}}{\partial x^{3}} \\
& +\frac{2 E t_{f}^{3}}{3\left(1-\mu^{2}\right)} \frac{\partial^{4} w}{\partial x^{4}}-\frac{E t_{f}^{2} h_{c}}{2\left(1-\mu^{2}\right)} \frac{\partial^{3} \phi_{y c}}{\partial y \partial x^{2}}-\sum_{i=1}^{m} \frac{E_{c} h_{c} t_{c}}{2\left(1+\mu_{c}\right)}\left(\frac{\partial \phi_{x c}}{\partial x}+\frac{\partial^{2} w}{\partial x^{2}}\right) \delta_{D}\left(y-y_{i}\right)- \\
& \sum_{j=1}^{n} \frac{E_{c} h_{c} t_{c}}{2\left(1+\mu_{c}\right)}\left(\frac{\partial \phi_{y c}}{\partial y}+\frac{\partial^{2} w}{\partial y^{2}}\right) \delta_{D}\left(x-x_{j}\right)-P(x, y, t)-N_{x} \frac{\partial^{2} w}{\partial x^{2}}-2 N_{x y} \frac{\partial^{2} w}{\partial x \partial y}-N_{y} \frac{\partial^{2} w}{\partial y^{2}}= \\
& -\frac{1}{2} \rho t_{f}^{2} h_{c}\left(\frac{\partial^{3} \phi_{x c}}{\partial x \partial t^{2}}+\frac{\partial^{3} \phi_{y c}}{\partial y \partial t^{2}}\right)-2 \rho t_{f} \frac{\partial^{2} w}{\partial t^{2}}+\frac{2 \rho t_{f}^{3}}{3}\left(\frac{\partial^{4} w}{\partial x^{2} \partial t^{2}}+\frac{\partial^{4} w}{\partial y^{2} \partial t^{2}}\right)-\sum_{i=1}^{m} \rho_{c} h_{c} t_{c} \frac{\partial^{2} w}{\partial t^{2}} \delta_{D}\left(y-y_{i}\right) \\
& -\sum_{j=1}^{n} \rho_{c} h_{c} t_{c} \frac{\partial^{2} w}{\partial t^{2}} \delta_{D}\left(x-x_{j}\right)
\end{aligned}
$$

where $\delta_{D}\left(x-x_{i}\right)$ and $\delta_{D}\left(y-y_{j}\right)$ are the Dirac Delta functions at the locations of core sheets spanning along $x$ - and $y$-axes respectively.

\section{Analytical solutions}

The present study focuses on the bending, global buckling and free vibration behaviour of simply supported sandwich panels with square honeycomb cores. This can be achieved by Equations (17) using the Galerkin approach. The boundary conditions of a simply supported rectangular sandwich panel can be expressed as follows:

(a) At edges $x=0$ and $x=a: \quad v_{o c}=0 ; \phi_{y c}=0 ; w=0$ 
(b) At edges $y=0$ and $y=b: \quad u_{o c}=0 ; \phi_{x c}=0 ; w=0$

The displacement variables satisfying the above boundary conditions can be expressed in terms of the unknown parameters $U_{p q}, V_{p q}, \phi_{x p q}, \phi_{y p q}$ and $W_{p q}$ as

$$
\begin{aligned}
& u_{o c}=\sum_{p=1}^{M} \sum_{q=1}^{N} U_{p q} \cos (p \pi x / a) \sin (q \pi y / b) e^{i \omega t} \\
& v_{o c}=\sum_{p=1}^{M} \sum_{q=1}^{N} V_{p q} \sin (p \pi x / a) \cos (q \pi y / b) e^{i \omega t} \\
& \phi_{x c}=\sum_{p=1}^{M} \sum_{q=1}^{N} \phi_{x p q} \cos (p \pi x / a) \sin (q \pi y / b) e^{i \omega t} \\
& \phi_{y c}=\sum_{p=1}^{M} \sum_{q=1}^{N} \phi_{y p q} \sin (p \pi x / a) \cos (q \pi y / b) e^{i \omega t} \\
& w=\sum_{p=1}^{M} \sum_{q=1}^{N} W_{p q} \sin (p \pi x / a) \sin (q \pi y / b) e^{i \omega t}
\end{aligned}
$$

where $\omega$ is the natural frequency, $M$ and $N$ are the prescribed numbers of modal waves in the $x$ - and $y$-directions.

\subsection{Bending analysis}

Setting $\omega=0$ in Eq. (19), substituting Eq. (19) into Eq. (17) and then using the Galerkin approach, one may get a set of algebraic equations in terms of the unknown parameters $U_{p q}$, $V_{p q}, \phi_{x p q}, \phi_{y p q}$ and $W_{p q}$. These equations can be expressed in matrix form as

$$
[\mathbf{K}]\{\Delta\}=\{\mathbf{F}\}
$$

where the generalized displacement vector $\{\boldsymbol{\Delta}\}$ and force vector $\{\mathbf{F}\}$ are given by

$$
\{\boldsymbol{\Delta}\}^{\mathrm{T}}=\left\{U_{p q}, V_{p q}, \phi_{x p q}, \phi_{y p q}, W_{p q}\right\} ;\{\mathbf{F}\}^{\mathrm{T}}=\left\{F_{1}, F_{2}, F_{3}, F_{4}, F_{5}\right\}
$$

and the elements of stiffness matrix $[\mathbf{K}]$ and force vector $\{\mathbf{F}\}$ are given in Appendix $A$. The generalized displacement vector $\{\boldsymbol{\Delta}\}$ can be obtained by solving Eq. (20).

\subsection{Buckling analysis}

To investigate the global buckling of sandwich panels subjected to external in-plane loads, one may set $P(x, y, t)=0$ in Eq. (17) and $\omega=0$ in Eq. (19). Substituting Eq. (19) into Eq. 
(17) and using the Galerkin method, the global buckling problem can be written in matrix form as

$$
([\mathbf{K}]-[\mathbf{Q}])\{\boldsymbol{\Delta}\}=\{\mathbf{0}\}
$$

where the elements of the matrix [Q] are also given in Appendix A. To obtain the non-trivial solution of Eq. (22) and hence the buckling loads, one may equate the determinant of matrix in the left hand side to zero, namely

$$
|[\mathbf{K}]-[\mathbf{Q}]|=0
$$

\subsection{Free vibration analysis}

For the free vibration case, one may similarly set $P(x, y, t)=0$ in Eq. (17). Substituting Eq. (19) into Eq. (17) and using the Galerkin method, the free vibration problem can be formulated as

$$
\left([\mathbf{K}]-\omega^{2}[\mathbf{M}]\right)\{\boldsymbol{\Delta}\}=\{\mathbf{0}\}
$$

where the elements of mass matrix $[\mathbf{M}]$ are given in Appendix A. To obtain the non-trivial solution of Eq. (24) and hence the natural frequencies, one may equate the determinant of matrix in the left hand side to zero, namely

$$
\left|[\mathbf{K}]-\omega^{2}[\mathbf{M}]\right|=0
$$

\section{Numerical results and discussion}

The bending, global buckling and free vibration responses of rectangular sandwich panels with square honeycomb cores simply supported on four edges are analyzed by the proposed method. The results are then compared with those obtained from 3D finite element analysis and available solutions in the literature.

\subsection{Bending problem}

For ease of comparison, the geometric and material parameters of the square-honeycomb sandwich panel are taken as those of Kapania et al. ${ }^{14}$ The sandwich panel dimensions are $200 \mathrm{~mm} \times 200 \mathrm{~mm}$ and the thickness of face sheets $t_{f}$ is $2 \mathrm{~mm}$. The core height $h_{c}$ is $6 \mathrm{~mm}$ and the thickness of core sheets $t_{c}$ is $0.8 \mathrm{~mm}$. Three schemes with different core relative 
densities $^{9} \rho_{r}=\left(2 L_{c} t_{c}-t_{c}^{2}\right) / L_{c}^{2} \approx 2 t_{c} / L_{c}$ are considered, which are $10 \%, 15 \%$ and $20 \%$ with the corresponding core sheet spacings $L_{c}$ of $16 \mathrm{~mm}, 10.67 \mathrm{~mm}$ and $8 \mathrm{~mm}$ respectively. The face sheets and honeycomb cores are all made of aluminium with Young's modulus of 69GPa and Poisson's ratio of 0.25 . An out-of-plane pressure of $1 \mathrm{MPa}$ is applied on the one face sheet.

In the analysis, transverse bending and shear of the core sheets may be considered or ignored. In order to study the contribution of transverse bending of the core sheets to the response of the sandwich panel, analysis is carried out for both cases. A convergence study for bending analysis is carried out by increasing the number of terms in the assumed displacement functions expressed by Eq. (19). The results for the case of $\rho_{r}=20 \%$ as shown in Table 1 indicate that the results converge fast. It is accurate enough to use only five terms of the displacement function, which implies that the present method is very efficient compared with finite element analysis. Therefore hereafter, only five terms in Eq. (19) are used unless otherwise stated. Closer examination of Table 1 indicates that the even terms of the displacement function actually do not contribute to the solution, as both the structure and loading are symmetric about the centrelines.

Table 2 compares the displacement at the centre $(0.5 a, 0.5 b)$ of sandwich panel for three different core relative densities, i.e. $10 \%, 15 \%$, and $20 \%$, with those given by Kapania et al. ${ }^{14}$ Figure 3 shows the present results for deflection at $x=100 \mathrm{~mm}, 50 \mathrm{~mm}, 26 \mathrm{~mm}$ for $\rho_{r}=20 \%$ compared with those from finite element analysis. It is observed that the maximum deflections obtained from the proposed semi-analytical method are very close to the reference for the three core relative densities. In particular, the semi-analytical results are almost identical to the ESL results given by Kapania et al. ${ }^{14}$ The present results have errors of 6\% approximately compared with results of the detailed 3D finite element analysis with much more degrees of freedom, which can much better model the shear deformation in the thickness direction. Table 2 also shows that the differences between results obtained with transverse bending of core sheets considered and neglected are very small, which implies that this bending effect has little influence on the response of the sandwich panels. This is also consistent with the conclusions drawn in relevant publications that the core of sandwich 
panels mainly resists shearing loads. Hereafter, the transverse bending of core sheets is considered unless otherwise stated.

\subsection{Global buckling analysis}

The sandwich panel is then subjected to a uniform in-plane pressure on the two opposite sides along $x$-direction. The geometric parameters of the panel are: $a=1000 \mathrm{~mm}, t_{f}=1 \mathrm{~mm}, h_{c}$ $=10 \mathrm{~mm}, L_{c}=10 \mathrm{~mm}, t_{c}=0.5 \mathrm{~mm}$ and $b$ is defined by the value of $a / b$. The core relative density is $10 \%$ while the other material properties are the same as those in Section 4.1. For comparison, a 3D finite element analysis is performed by using the software ANSYS. The face and core sheets are modelled using the Shell63 elements and fine meshes (up to two elements per cell in $x$ - or $y$-direction and 4 elements in the thickness direction) are employed to ensure convergence of the calculated results. The critical buckling loads obtained by the present method agree well with those from 3D finite element analysis as shown in Table 3, with relative errors less than $5 \%$.

For ease of comparison, the calculated buckling loads are normalized by the corresponding minimum buckling load for each case. Figures 4 and 5 present the normalized buckling loads against the ratio of core height to sandwich panel height $h_{c} / h$ and the ratio of core sheet spacing to core height $L_{c} / h_{c}$, respectively, for panels with $a / b=1$. In Figure 4, only the core height $h_{c}$ and the thickness $t_{f}$ of the face sheet are varied with the other parameters unchanged. Similarly in Figure 5, only the core sheet spacing $L_{c}$ is varied while the other parameters are fixed and $h_{c} / h=0.8$. As seen in Figure 4 , the core height has significant effect on the global buckling load. As the ratio $h_{c} / h$ decreases while the total thickness $h$ is kept fixed, which implies an increase in total sandwich panel stiffness, the global buckling loads increase. For example, the global buckling load increases more than 4 times when the ratio $h_{c} / h$ reduces from 0.9 to 0.4 . By contrast, the core sheet spacing $L_{c}$ has different effect on the global buckling loads depending on the 
total panel height $h$. The effect becomes more obvious as the total panel height increases as shown in Figure 5 . On the other hand, the global buckling load only increases by approximately 5\% when the ratio $L_{c} / h_{c}$ decreases from 2 to 0.5 for the case of $h=12 \mathrm{~mm}$. However for the case of $h=50 \mathrm{~mm}$, the global buckling load increases by more than $60 \%$ for the same change in the ratio $L_{c} / h_{c}$. This is because as the core height increases, the shear of core sheets becomes more significant, thereby contributing more to the stiffness of the sandwich panels. These results indicate that the core height has more infuence on the global stiffness of the sandwich panels than the core sheet spacing.

\subsection{Free vibration analysis}

The example used in Section 4.2 is further analyzed for its free vibration. The material density of the face and core sheets is $2770 \mathrm{~kg} / \mathrm{m}^{3}$. Table 4 compares the first 5 natural frequencies of sandwich panels of different dimensions obtained from the proposed method with those from 3D finite element analysis using the same arrangements as in Section 4.2. Good agreement is observed. In order to investigate the effects of the core height and core sheet spacing on the first natural frequency of the sandwich panel, further calculations are carried out for the case $a / b=1$ with different sandwich panel heights. For ease of comparison, the calculated frequencies are normalized by the corresponding maximum frequency for each case.

Figures 6 and 7 present the normalized first natural frequencies against the ratio of core height to sandwich panel height $h_{c} / h$ and the ratio of core sheet spacing to core height $L_{c} / h_{c}$, respectively, for panels with $a / b=1$. Figure 6 shows all the curves have roughly the same summit shape as the ratio $h_{c} / h$ increases, with the first natural frequency reaching a maximum around $h_{c} / h \cong 0.75$ to 0.78 . As the ratio $h_{c} / h$ of core height to total panel height increases, the face sheet thickness decreases resulting in a decrease in stiffness and hence tending to lower the natural frequency. However at the same time, the sandwich panel is also getting lighter which tends to increase the natural frequency. Summit curves have therefore resulted from the combined effects of two opposing trends. Figure 7 shows that, 
for the common range of ratio $L_{c} / h_{c}$ from 0.4 to 2.0, the core sheet spacing $L_{c}$ has different effects on the first natural frequencies for different total panel heights. As seen in the variation of the first frequency of the sandwich panel against $L_{c} / h_{c}$ in Figure 7 , the cases of $h=12 \mathrm{~mm}$ and $h=20 \mathrm{~mm}$ show a monotonic increasing trend, the case of $h=50 \mathrm{~mm}$ displays a monotonic decreasing trend and the cases of $h=30 \mathrm{~mm}$ and $h=40 \mathrm{~mm}$ are of summit shapes. As the core sheet spacing increases, the effect of the sandwich panel mass on the frequencies is predominant for those with small total panel height, such as the case of $h$ $=12 \mathrm{~mm}$. When the total panel height increases, the effects of the sandwich panel stiffness due to variation of the core sheet spacing on the frequencies is predominant, such as the case of $h=50 \mathrm{~mm}$. Between the two extremes, both the effects of sandwich panel mass and stiffness on the frequencies are significant, as seen in Figure 7 for the cases of $h=30 \mathrm{~mm}$ and $h=40 \mathrm{~mm}$.

\section{Conclusions}

A semi-analytical method has been developed for the bending, global buckling and free vibration analyses of sandwich panels with square honeycomb cores. Using Hamilton's principle for formulation of the governing equations and the Galerkin approach for solution, close-form solutions for the simply supported condition are obtained. Comparison with the results of 3D finite element analysis and available results in the literature confirms that the assumptions made are reasonable and the accuracy of the proposed method is very good.

The present method accounts for the discrete nature of the core by treating the square-honeycomb sandwich panels as composite structures of plates and beams so that all the geometric and material parameters are included in the analysis. The method not only provides an accurate and efficient tool for predicting the global performance of sandwich panels with square-honeycomb core such as bending, global buckling and free vibration, but also enables optimal design of sandwich panels to be carried out conveniently.

The parameter study shows that the core height has more influence on the stiffness of the panels than the core sheet spacing. The effect of core sheet spacing on the stiffness of sandwich panels is different for different core heights. The bigger the core height is, the 
bigger is the influence on stiffness. Both the stiffness and mass of the sandwich panel change with variation of the core height and core sheet spacing. It implies that, where the natural frequencies of sandwich panels are at stake, more attention should be paid to the effects of the core height and core sheet spacing.

\section{References}

1. A. K. Noor and W. S. Burton, Computational models for sandwich panels and shells, Applied Mechanics Reviews. 49(3) (1996) 155-199.

2. Y. Frostig, Classical and high-order computational models in the analysis of modern sandwich panels, Composite Part B: Engineering 34(1) (2003) 83-100.

3. C. Libove and R. E. Hubka, Elastic constants for corrugated-core sandwich plates, NACA TN2289, Langley Aeronautical Laboratory, Langley Field, VA, 1951.

4. J. Penzien and T. Didriksson, Effective shear modulus of honeycomb cellular structure, AIAA Journal 2(3) (1964) 531-535.

5. L. J. Gibson and M. F. Ashby, Cellular solids: structure and properties, Pergamon, Oxford (1988).

6. M. Grediac, A finite element study of the transverse shear in honeycomb cores, International Journal of Solids and Structures 30(13) (1993) 1777-1788.

7. T. C. Fung, K. H. Tan and T. S. Lok, Elastic constants for Z-core sandwich panels, Journal of Structural Engineering 120(10) (1994) 3046-3055.

8. S. Aimmanee and J. R. Vinson, Analysis and optimization of foam-reinforced web core composite sandwich panels under in-plane compressive loads, Journal of Sandwich Structures and Materials 4(2) (2002) 115-139.

9. S. A. Javachandran, A. Soundararajan, S. Seetharaman and G. M. S. Knight, Modulus of core reaction approach to buckling of sandwich plates, International Journal of Structural Stability and Dynamics 4(4) (2004) 579-588.

10. J. Romanoff and P. Varsta, Bending response of web-core sandwich beams, Composite Structures 73(4) (2006) 478-487.

11. J. Romanoff and P. Varsta, Bending response of web-core sandwich plates, Composite Structures 81(2) (2007) 292-302. 
12. F. W. Zok, S. A. Waltner, Z. Wei, H. J. Rathbun, R. M. McMeeking and A. G. Evans, A protocol for characterizing the structural performance of metallic sandwich panels: application to pyramidal truss cores, International Journal of Solid and Structures 41(22-23) (2004) 6249-6271.

13. T. Rabczuk, J. Y. Kim, E. Samaniego and T. Belytschko, Homogenization of sandwich structures, International Journal for Numerical Methods in Engineering 61(7) (2004) 1009-1027.

14. R. K. Kapania, H. E. Soliman, S. Vasudeva, O. Hughes and D. P. Makhecha, Static analysis of sandwich panels with square honeycomb core, AIAA Journal, 46(3) (2008) 627-634.

15. N. Buannic, P. Cartraud and T. Quesnel, Homogenization of corrugated core sandwich panels, Composite Structures 59(3) (2003) 299-312.

16. Z. Y. Xue and J. W. Hutchinson, A comparative study of impulse-resistant metal sandwich plates, International Journal of Impact Engineering 30(10) (2004) 1283-1305.

17. G. J. McShane, D. D. Radford, V. S. Deshpande and N. A. Fleck, The response of clamped sandwich plates with lattice cores subjected to shock loading, European Journal of Mechanics, A/Solids 25(2) (2006) 215-229.

18. F. Cote, V. S. Deshpande, N. A. Fleck and A. G. Evans, The out-of-plane compressive behavior of metallic honeycombs, Materials Science and Engineering A 380(1-2) (2004) 272-280.

19. Z. Y. Xue and J. W. Hutchinson, Constitutive model for quasi-static deformation of metallic sandwich cores, International Journal for Numerical Methods in Engineering 61(13) (2004) 2205-2238.

20. Z. Y. Xue, A. Vaziri and J. W. Hutchinson, Non-uniform hardening constitutive model for compressible orthotropic materials with application to sandwich plate cores, CMES-computer Modeling in Engineering and Sciences 10(1) (2005) 79-95.

21. F. W. Zok, H. Rathbun, M. He, E. Ferri, C. Mercer, R. M. Mcmeeking and A. G. Evans, Structural performance of metallic sandwich panels with square honeycomb cores, Philosophical Magazine 85(26-27) (2005) 3207-3234.

22. Q. H. Cheng, H. P. Lee and C. Lu, A numerical analysis approach for evaluating elastic 
constants of sandwich structures with various cores, Composite Structures 74(2) (2006) 226-236.

23. H. J. Rathbun, Z. Wei, M. Y. He, F. W. Zok, A. G. Evans, D. J. Sypeck and H. N. G. Wadley, Measurement and simulation of the performance of a lightweight metallic sandwich structure with a tetrahedral truss core, Journal of Applied Mechanics, Transactions ASME 71(3) (2004) 368-374.

24. T. S. Lok, Bending and forced vibration response of a clamped orthotropic thick plate and sandwich panel, Journal of Sound and Vibration 245(1) (2001) 63-78.

25. W. S. Burton and A. K. Noor, Assessment of continuum models for sandwich panels honeycomb cores, Computer Methods in Applied Mechanics and Engineering 145(3-4) (1997) 341-360.

26. X. F. Xu, P. Qiao, Homogenized elastic properties of honeycomb sandwich with skin effect, International Journal of Solids and Structures 39(8) (2002) 2153-2188.

\section{Appendix A}

Let $M$ and $N$ be the numbers of trigonometric functions used to define the displacement functions in Eq. (19). The matrix [K] can be written as

$$
[\mathbf{K}]=\left[\begin{array}{ccccc}
{\left[\mathbf{K}_{11}\right]_{M N \times M N}} & {\left[\mathbf{K}_{12}\right]_{M N \times M N}} & {\left[\mathbf{K}_{13}\right]_{M N \times M N}} & {\left[\mathbf{K}_{14}\right]_{M N \times M N}} & {\left[\mathbf{K}_{15}\right]_{M N \times M N}} \\
& {\left[\mathbf{K}_{22}\right]_{M N \times M N}} & {\left[\mathbf{K}_{23}\right]_{M N \times M N}} & {\left[\mathbf{K}_{24}\right]_{M N \times M N}} & {\left[\mathbf{K}_{25}\right]_{M N \times M N}} \\
& & {\left[\mathbf{K}_{33}\right]_{M N \times M N}} & {\left[\mathbf{K}_{34}\right]_{M N \times M N}} & {\left[\mathbf{K}_{35}\right]_{M N \times M N}} \\
& & & {\left[\mathbf{K}_{44}\right]_{M N \times M N}} & {\left[\mathbf{K}_{45}\right]_{M N \times M N}} \\
\text { symm. } & & & & {\left[\mathbf{K}_{55}\right]_{M N \times M N}}
\end{array}\right]
$$

where $M N$ denotes the product of $M$ and $N$, and $\left[\mathbf{K}_{i j}\right]$ is an $M N \times M N$ submatrix. The elements $K_{i j}(I, J)$ of submatrix $\left[\mathbf{K}_{i j}\right]$ are given by

$$
\begin{aligned}
& K_{11}((r-1) \cdot N+s,(p-1) \cdot N+q)= \\
& \int_{0}^{b} \int_{0}^{a}\left[\left(\frac{2 E t_{f}}{1-\mu^{2}} \frac{p^{2} \pi^{2}}{a^{2}}+\frac{E t_{f}}{1+\mu} \frac{q^{2} \pi^{2}}{b^{2}}\right) \cos (p \pi x / a) \sin (q \pi y / b)\right][\cos (r \pi x / a) \sin (s \pi y / b)] d x d y+ \\
& \int_{0}^{b} \int_{0}^{a}\left[\sum_{i=1}^{m} E_{c} h_{c} t_{c} \frac{p^{2} \pi^{2}}{a^{2}} \cos (p \pi x / a) \sin (q \pi y / b) \delta_{D}\left(y-y_{i}\right)\right][\cos (r \pi x / a) \sin (s \pi y / b)] d x d y \\
& \quad(p, r=1,2,3 \ldots M \quad q, s=1,2,3 \ldots N)
\end{aligned}
$$




$$
\begin{aligned}
& K_{12}((r-1) \cdot N+s,(p-1) \cdot N+q)= \\
& \int_{0}^{b} \int_{0}^{a}\left[\frac{E t_{f}}{1-\mu} \frac{p q \pi^{2}}{a b} \cos (p \pi x / a) \sin (q \pi y / b)\right][\cos (r \pi x / a) \sin (s \pi y / b)] d x d y
\end{aligned}
$$

$$
\begin{aligned}
& K_{13}((r-1) \cdot N+s,(p-1) \cdot N+q)=0 \\
& K_{14}((r-1) \cdot N+s,(p-1) \cdot N+q)=0 \\
& K_{15}((r-1) \cdot N+s,(p-1) \cdot N+q)=0
\end{aligned}
$$

$K_{22}((r-1) \cdot N+s,(p-1) \cdot N+q)=$

$\int_{0}^{b} \int_{0}^{a}\left[\left(\frac{2 E t_{f}}{1-\mu^{2}} \frac{q^{2} \pi^{2}}{b^{2}}+\frac{E t_{f}}{1+\mu} \frac{p^{2} \pi^{2}}{a^{2}}\right) \sin (p \pi x / a) \cos (q \pi y / b)\right][\sin (r \pi x / a) \cos (s \pi y / b)] d x d y+$ $\int_{0}^{b} \int_{0}^{a}\left[\sum_{j=1}^{n} E_{c} h_{c} t_{c} \frac{q^{2} \pi^{2}}{b^{2}} \sin (p \pi x / a) \cos (q \pi y / b) \delta_{D}\left(x-x_{j}\right)\right][\sin (r \pi x / a) \cos (s \pi y / b)] d x d y$

$$
\begin{aligned}
& K_{23}((r-1) \cdot N+s,(p-1) \cdot N+q)=0 \\
& K_{24}((r-1) \cdot N+s,(p-1) \cdot N+q)=0 \\
& K_{25}((r-1) \cdot N+s,(p-1) \cdot N+q)=0
\end{aligned}
$$

$K_{33}((r-1) \cdot N+s,(p-1) \cdot N+q)=$

$\int_{0}^{b} \int_{0}^{a}\left[\left(\frac{E t_{f} h_{c}^{2}}{2\left(1-\mu^{2}\right)} \frac{p^{2} \pi^{2}}{a^{2}}+\frac{E t_{f} h_{c}^{2}}{4(1+\mu)} \frac{q^{2} \pi^{2}}{b^{2}}\right) \cos (p \pi x / a) \sin (q \pi y / b)\right][\cos (r \pi x / a) \sin (s \pi y / b)] d x d y+$ $\int_{0}^{b} \int_{0}^{a}\left[\sum_{i=1}^{m}\left(\frac{E_{c} h_{c} t_{c}}{2\left(1+\mu_{c}\right)}+\frac{E_{c} h_{c}^{3} t_{c}}{12} \frac{p^{2} \pi^{2}}{a^{2}}\right) \cos (p \pi x / a) \sin (q \pi y / b) \delta_{D}\left(y-y_{i}\right)\right][\cos (r \pi x / a) \sin (s \pi y / b)] d x d y$

$$
\begin{aligned}
& K_{34}((r-1) \cdot N+s,(p-1) \cdot N+q)= \\
& \int_{0}^{b} \int_{0}^{a}\left[\frac{E t_{f} h_{c}^{2}}{4(1-\mu)} \frac{p q \pi^{2}}{a b} \cos (p \pi x / a) \sin (q \pi y / b)\right][\cos (r \pi x / a) \sin (s \pi y / b)] d x d y
\end{aligned}
$$

$K_{35}((r-1) \cdot N+s,(p-1) \cdot N+q)=$

$\int_{0}^{b} \int_{0}^{a}\left[-\frac{E t_{f}^{2} h_{c}}{2\left(1-\mu^{2}\right)}\left(\frac{p^{3} \pi^{3}}{a^{3}}+\frac{p q^{2} \pi^{3}}{a b^{2}}\right) \cos (p \pi x / a) \sin (q \pi y / b)\right][\cos (r \pi x / a) \sin (s \pi y / b)] d x d y+$ $\int_{0}^{b} \int_{0}^{a}\left[\sum_{i=1}^{m} \frac{E_{c} h_{c} t_{c}}{2\left(1+\mu_{c}\right)} \frac{m \pi}{a} \cos (p \pi x / a) \sin (q \pi y / b) \delta_{D}\left(y-y_{i}\right)\right][\cos (r \pi x / a) \sin (s \pi y / b)] d x d y$ $K_{44}((r-1) \cdot N+s,(p-1) \cdot N+q)=$ $\int_{0}^{b} \int_{0}^{a}\left[\left(\frac{E t_{f} h_{c}^{2}}{2\left(1-\mu^{2}\right)} \frac{q^{2} \pi^{2}}{b^{2}}+\frac{E t_{f} h_{c}^{2}}{4(1+\mu)} \frac{p^{2} \pi^{2}}{a^{2}}\right) \sin (p \pi x / a) \cos (q \pi y / b)\right][\sin (r \pi x / a) \cos (s \pi y / b)] d x d y+$ $\int_{0}^{b} \int_{0}^{a}\left[\sum_{j=1}^{n}\left(\frac{E_{c} h_{c} t_{c}}{2\left(1+\mu_{c}\right)}+\frac{E_{c} h_{c}^{3} t_{c}}{12} \frac{q^{2} \pi^{2}}{b^{2}}\right) \sin (p \pi x / a) \cos (q \pi y / b) \delta_{D}\left(x-x_{j}\right)\right][\sin (r \pi x / a) \cos (s \pi y / b)] d x d y$ 


$$
\begin{aligned}
& K_{45}((r-1) \cdot N+s,(p-1) \cdot N+q)= \\
& \int_{0}^{b} \int_{0}^{a}\left[-\frac{E t_{f}^{2} h_{c}}{2\left(1-\mu^{2}\right)}\left(\frac{q^{3} \pi^{3}}{b^{3}}+\frac{p^{2} q \pi^{3}}{a^{2} b}\right) \sin (p \pi x / a) \cos (q \pi y / b)\right][\sin (r \pi x / a) \cos (s \pi y / b)] d x d y+ \\
& \int_{0}^{b} \int_{0}^{a}\left[\sum_{j=1}^{n} \frac{E_{c} h_{c} t_{c}}{2\left(1+\mu_{c}\right)} \frac{n \pi}{b} \sin (p \pi x / a) \cos (q \pi y / b) \delta_{D}\left(x-x_{j}\right)\right][\sin (r \pi x / a) \cos (s \pi y / b)] d x d y \\
& K_{55}((r-1) \cdot N+s,(p-1) \cdot N+q)= \\
& \int_{0}^{b} \int_{0}^{a}\left[\frac{2 E t_{f}^{3} \pi^{4}}{3\left(1-\mu^{2}\right)}\left(\frac{2 p^{2} q^{2}}{a^{2} b^{2}}+\frac{p^{4}}{a^{4}}+\frac{q^{4}}{b^{4}}\right) \sin (p \pi x / a) \sin (q \pi y / b)\right][\sin (r \pi x / a) \sin (s \pi y / b)] d x d y+ \\
& \int_{0}^{b} \int_{0}^{a}\left[\sum_{i=1}^{m} \frac{E_{c} h_{c} t_{c}}{2\left(1+\mu_{c}\right)} \frac{p^{2} \pi^{2}}{a^{2}} \sin (p \pi x / a) \sin (q \pi y / b) \delta_{D}\left(y-y_{i}\right)\right][\sin (r \pi x / a) \sin (s \pi y / b)] d x d y \\
& \int_{0}^{b} \int_{0}^{a}\left[\sum_{i=1}^{n} \frac{E_{c} h_{c} t_{c}}{2\left(1+\mu_{c}\right)} \frac{q^{2} \pi^{2}}{b^{2}} \sin (p \pi x / a) \sin (q \pi y / b) \delta_{D}\left(x-x_{j}\right)\right][\sin (r \pi x / a) \sin (s \pi y / b)] d x d y
\end{aligned}
$$

The force vector $[\mathbf{F}]$ can be written as

$$
\left.[\mathbf{F}]=\left[\begin{array}{lllll}
{\left[\mathbf{F}_{1}\right]_{1 \times M N}} & {\left[\mathbf{F}_{2}\right]_{1 \times M N}} & {\left[\mathbf{F}_{3}\right]_{1 \times M N}} & {\left[\mathbf{F}_{4}\right]_{1 \times M N}} & {\left[\mathbf{F}_{5}\right.}
\end{array}\right]_{1 \times M N}\right]^{\mathrm{T}}
$$

where the elements of the row vector $\left[\mathbf{F}_{i}\right]$ are given below

$$
\begin{gathered}
F_{1}(1,(r-1) \cdot N+s)=0 \\
F_{2}(1,(r-1) \cdot N+s)=0 \\
F_{3}(1,(r-1) \cdot N+s)=0 \\
F_{4}(1,(r-1) \cdot N+s)=0 \\
F_{5}(1,(r-1) \cdot N+s)=\int_{0}^{b} \int_{0}^{a} P(x, y) \sin (r \pi x / a) \sin (s \pi y / b) d x d y
\end{gathered}
$$

The matrix $[\mathbf{Q}]$ is written as

$$
[\boldsymbol{Q}]=\left[\begin{array}{ccccc}
{\left[\boldsymbol{Q}_{11}\right]_{M N \times M N}} & {\left[\boldsymbol{Q}_{12}\right]_{M N \times M N}} & {\left[\boldsymbol{Q}_{13}\right]_{M N \times M N}} & {\left[\boldsymbol{Q}_{14}\right]_{M N \times M N}} & {\left[\boldsymbol{Q}_{15}\right]_{M N \times M N}} \\
& {\left[\boldsymbol{Q}_{22}\right]_{M N \times M N}} & {\left[\boldsymbol{Q}_{23}\right]_{M N \times M N}} & {\left[\boldsymbol{Q}_{24}\right]_{M N \times M N}} & {\left[\boldsymbol{Q}_{25}\right]_{M N \times M N}} \\
& & {\left[\boldsymbol{Q}_{33}\right]_{M N \times M N}} & {\left[\boldsymbol{Q}_{34}\right]_{M N \times M N}} & {\left[\boldsymbol{Q}_{35}\right]_{M N \times M N}} \\
& & & {\left[\boldsymbol{Q}_{44}\right]_{M N \times M N}} & {\left[\boldsymbol{Q}_{45}\right]_{M N \times M N}} \\
\text { symm. } & & & & {\left[\boldsymbol{Q}_{55}\right]_{M N \times M N}}
\end{array}\right]
$$

where the elements $Q_{i j}(I, J)$ of sub-matrix $\left[\boldsymbol{Q}_{i j}\right]$ are zero except for sub-matrix $\left[\boldsymbol{Q}_{55}\right]$, which has elements given as 
$Q_{55}((r-1) \cdot N+s,(p-1) \cdot N+q)=$

$\int_{0}^{b} \int_{0}^{a}\left[-N_{x} \frac{p^{2} \pi^{2}}{a^{2}} \sin (p \pi x / a) \sin (q \pi y / b)\right][\sin (r \pi x / a) \sin (s \pi y / b)] d x d y+$

$\int_{0}^{b} \int_{0}^{a}\left[N_{x y} \frac{p q \pi^{2}}{a b} \cos (p \pi x / a) \cos (q \pi y / b)\right][\sin (r \pi x / a) \sin (s \pi y / b)] d x d y+$

$\int_{0}^{b} \int_{0}^{a}\left[-N_{y} \frac{q^{2} \pi^{2}}{b^{2}} \sin (p \pi x / a) \sin (q \pi y / b)\right][\sin (r \pi x / a) \sin (s \pi y / b)] d x d y$

The mass matrix $[\mathbf{M}]$ is written as

$$
[\mathbf{M}]=\left[\begin{array}{ccccc}
{\left[\mathbf{M}_{11}\right]_{M N \times M N}} & {\left[\mathbf{M}_{12}\right]_{M N \times M N}} & {\left[\mathbf{M}_{13}\right]_{M N \times M N}} & {\left[\mathbf{M}_{14}\right]_{M N \times M N}} & {\left[\mathbf{M}_{15}\right]_{M N \times M N}} \\
& {\left[\mathbf{M}_{22}\right]_{M N \times M N}} & {\left[\mathbf{M}_{23}\right]_{M N \times M N}} & {\left[\mathbf{M}_{24}\right]_{M N \times M N}} & {\left[\mathbf{M}_{25}\right]_{M N \times M N}} \\
& & {\left[\mathbf{M}_{33}\right]_{M N \times M N}} & {\left[\mathbf{M}_{34}\right]_{M N \times M N}} & {\left[\mathbf{M}_{35}\right]_{M N \times M N}} \\
& & & {\left[\mathbf{M}_{44}\right]_{M N \times M N}} & {\left[\mathbf{M}_{45}\right]_{M N \times M N}} \\
\text { symm. } & & & {\left[\mathbf{M}_{55}\right]_{M N \times M N}}
\end{array}\right]
$$

with the sub-matrix $\left[M_{i j}\right]$ having elements $M_{i j}(I, J)$ given as

$M_{11}((r-1) \cdot N+s,(p-1) \cdot N+q)=$

$\int_{0}^{b} \int_{0}^{a}\left[2 \rho t_{f} \cos (p \pi x / a) \sin (q \pi y / b)\right][\cos (r \pi x / a) \sin (s \pi y / b)] d x d y+$

$\int_{0}^{b} \int_{0}^{a}\left[\sum_{i=1}^{m} \rho_{c} h_{c} t_{c} \cos (p \pi x / a) \sin (q \pi y / b) \delta_{D}\left(y-y_{i}\right)\right][\cos (r \pi x / a) \sin (s \pi y / b)] d x d y$

$+\int_{0}^{b} \int_{0}^{a}\left[\sum_{j=1}^{n} \rho_{c} h_{c} t_{c} \cos (p \pi x / a) \sin (q \pi y / b) \delta_{D}\left(x-x_{j}\right)\right][\cos (r \pi x / a) \sin (s \pi y / b)] d x d y$

$$
\begin{aligned}
& M_{12}((r-1) \cdot N+s,(p-1) \cdot N+q)=0 \\
& M_{13}((r-1) \cdot N+s,(p-1) \cdot N+q)=0 \\
& M_{14}((r-1) \cdot N+s,(p-1) \cdot N+q)=0 \\
& M_{15}((r-1) \cdot N+s,(p-1) \cdot N+q)=0
\end{aligned}
$$

$M_{22}((r-1) \cdot N+s,(p-1) \cdot N+q)=$

$\int_{0}^{b} \int_{0}^{a}\left[2 \rho t_{f} \sin (p \pi x / a) \cos (q \pi y / b)\right][\sin (r \pi x / a) \cos (s \pi y / b)] d x d y+$

$\int_{0}^{b} \int_{0}^{a}\left[\sum_{j=1}^{n} \rho_{c} h_{c} t_{c} \sin (p \pi x / a) \cos (q \pi y / b) \delta_{D}\left(x-x_{j}\right)\right][\sin (r \pi x / a) \cos (s \pi y / b)] d x d y$

$+\int_{0}^{b} \int_{0}^{a}\left[\sum_{i=1}^{m} \rho_{c} h_{c} t_{c} \sin (p \pi x / a) \cos (q \pi y / b) \delta_{D}\left(y-y_{i}\right)\right][\sin (r \pi x / a) \cos (s \pi y / b)] d x d y$

$$
M_{23}((r-1) \cdot N+s,(p-1) \cdot N+q)=0
$$




$$
\begin{aligned}
& M_{24}((r-1) \cdot N+s,(p-1) \cdot N+q)=0 \\
& M_{25}((r-1) \cdot N+s,(p-1) \cdot N+q)=0
\end{aligned}
$$

$M_{33}((r-1) \cdot N+s,(p-1) \cdot N+q)=$

$\int_{0}^{b} \int_{0}^{a}\left[\frac{1}{2} \rho t_{f} h_{c}^{2} \cos (p \pi x / a) \sin (q \pi y / b)\right][\cos (r \pi x / a) \sin (s \pi y / b)] d x d y+$

$\int_{0}^{b} \int_{0}^{a}\left[\sum_{i=1}^{m} \rho_{c} \frac{h_{c}^{3}}{12} t_{c} \cos (p \pi x / a) \sin (q \pi y / b) \delta_{D}\left(y-y_{i}\right)\right][\cos (r \pi x / a) \sin (s \pi y / b)] d x d y$

$+\int_{0}^{b} \int_{0}^{a}\left[\sum_{j=1}^{n} \rho_{c} \frac{h_{c}^{3}}{12} t_{c} \cos (p \pi x / a) \sin (q \pi y / b) \delta_{D}\left(x-x_{j}\right)\right][\cos (r \pi x / a) \sin (s \pi y / b)] d x d y$

$$
M_{34}((r-1) \cdot N+s,(p-1) \cdot N+q)=0
$$

$$
M_{35}((r-1) \cdot N+s,(p-1) \cdot N+q)=
$$

$\int_{0}^{b} \int_{0}^{a}\left[-\frac{1}{2} \rho t_{f}^{2} h_{c} \frac{p \pi}{a} \cos (p \pi x / a) \sin (q \pi y / b)\right][\cos (r \pi x / a) \sin (s \pi y / b)] d x d y$

$M_{44}((r-1) \cdot N+s,(p-1) \cdot N+q)=$

$\int_{0}^{b} \int_{0}^{a}\left[\frac{1}{2} \rho t_{f} h_{c}^{2} \sin (p \pi x / a) \cos (q \pi y / b)\right][\sin (r \pi x / a) \cos (s \pi y / b)] d x d y+$ $\int_{0}^{b} \int_{0}^{a}\left[\sum_{j=1}^{n} \rho_{c} \frac{h_{c}^{3}}{12} t_{c} \sin (p \pi x / a) \cos (q \pi y / b) \delta_{D}\left(x-x_{j}\right)\right][\sin (r \pi x / a) \cos (s \pi y / b)] d x d y$ $+\int_{0}^{b} \int_{0}^{a}\left[\sum_{i=1}^{m} \rho_{c} \frac{h_{c}^{3}}{12} t_{c} \sin (p \pi x / a) \cos (q \pi y / b) \delta_{D}\left(y-y_{i}\right)\right][\sin (r \pi x / a) \cos (s \pi y / b)] d x d y$ $M_{45}((r-1) \cdot N+s,(p-1) \cdot N+q)=$ $\int_{0}^{b} \int_{0}^{a}\left[-\frac{1}{2} \rho t_{f}^{2} h_{c} \frac{q \pi}{b} \sin (p \pi x / a) \cos (q \pi y / b)\right][\sin (r \pi x / a) \cos (s \pi y / b)] d x d y$ $M_{55}((r-1) \cdot N+s,(p-1) \cdot N+q)=$ $\int_{0}^{b} \int_{0}^{a}\left[2 \rho t_{f}\left(1+\frac{p^{2} \pi^{2}}{3 a^{2}}+\frac{q^{2} \pi^{2}}{3 b^{2}}\right) \sin (p \pi x / a) \sin (q \pi y / b)\right][\sin (r \pi x / a) \sin (s \pi y / b)] d x d y+$ $\int_{0}^{b} \int_{0}^{a}\left[\sum_{i=1}^{m} \rho_{c} h_{c} t_{c} \sin (p \pi x / a) \sin (q \pi y / b) \delta_{D}\left(y-y_{i}\right)\right][\sin (r \pi x / a) \sin (s \pi y / b)] d x d y+$ $\int_{0}^{b} \int_{0}^{a}\left[\sum_{j=1}^{n} \rho_{c} h_{c} t_{c} \sin (p \pi x / a) \sin (q \pi y / b) \delta_{D}\left(x-x_{j}\right)\right][\sin (r \pi x / a) \sin (s \pi y / b)] d x d y$ 
Table 1. Convergence study for the displacements at centre of sandwich panel

\begin{tabular}{|c|c|c|c|}
\hline \multirow{2}{*}{$\begin{array}{c}\text { Case } \\
\text { No. }\end{array}$} & No. of terms in Eq. (19) & \multicolumn{2}{|c|}{ Displacement (mm) } \\
\cline { 3 - 4 } & $(M \times N)$ & $\begin{array}{c}\text { Transverse bending of } \\
\text { core sheets considered }\end{array}$ & $\begin{array}{r}\text { Transverse bending of } \\
\text { core sheets not considered }\end{array}$ \\
\hline 1 & $1 \times 1$ & 1.475 & 1.491 \\
\hline 2 & $2 \times 2$ & 1.475 & 1.491 \\
\hline 3 & $3 \times 3$ & 1.427 & 1.443 \\
\hline 4 & $4 \times 4$ & 1.427 & 1.443 \\
\hline 5 & $5 \times 5$ & 1.433 & 1.449 \\
\hline 6 & $6 \times 6$ & 1.433 & 1.449 \\
\hline 7 & $7 \times 7$ & 1.431 & 1.447 \\
\hline 8 & $8 \times 8$ & 1.431 & 1.448 \\
\hline 9 & $9 \times 9$ & 1.432 & 1.448 \\
\hline 10 & $10 \times 10$ & 1.432 & \\
\hline
\end{tabular}

Table 2. Displacement at centre of sandwich panel using different methods

\begin{tabular}{|c|c|c|c|c|c|c|c|}
\hline \multirow{2}{*}{$\rho_{r}$} & \multicolumn{5}{|c|}{ Displacement (mm) } \\
\cline { 2 - 8 } & \multicolumn{2}{|c|}{ Present method } & \multicolumn{4}{|c|}{ Kapania et al. } \\
\cline { 2 - 8 } & $\begin{array}{c}\text { Bending of } \\
\text { core sheets } \\
\text { considered }\end{array}$ & $\begin{array}{c}\text { Bending of } \\
\text { core sheets not } \\
\text { considered }\end{array}$ & $\begin{array}{c}\text { ABAQUS } \\
\text { detailed } \\
\text { model }\end{array}$ & ESL & \multicolumn{3}{|c|}{ Equivalent plate theory } \\
\cline { 3 - 8 } & 1.538 & 1.545 & 1.625 & 1.540 & - & - & - \\
\hline $10 \%$ & 1.469 & 1.481 & 1.560 & 1.472 & - & - & - \\
\hline $15 \%$ & 1.433 & 1.449 & 1.528 & 1.435 & 1.291 & 1.295 & 1.412 \\
\hline
\end{tabular}

Table 3. Global buckling loads (MPa) of sandwich panel using different methods

\begin{tabular}{|c|c|c|c|}
\hline$a / b$ & Present method & Finite element method & Error (\%) \\
\hline 1.0 & 16.2 & 16.5 & 1.9 \\
\hline 1.25 & 26.6 & 27.0 & 1.5 \\
\hline 2.0 & 63.5 & 66.1 & 4.1 \\
\hline
\end{tabular}


Table 4. Natural frequencies of sandwich panels using different methods

\begin{tabular}{|c|c|c|c|c|c|c|}
\hline \multirow{3}{*}{$a / b$} & & \multicolumn{5}{|c|}{ Natural frequencies (Hz) } \\
\cline { 3 - 7 } & & First & Second & Third & Fourth & Fifth \\
\hline \multirow{3}{*}{1.0} & Present method & 73.8 & 184.6 & 184.6 & 292.5 & 368.1 \\
\cline { 2 - 7 } & Finite element & 72.2 & 181.7 & 181.7 & 286.1 & 363.3 \\
\cline { 2 - 7 } & Error (\%) & 2.2 & 1.6 & 1.6 & 2.2 & 1.3 \\
\hline \multirow{3}{*}{1.25} & Present method & 93.9 & 203.6 & 262.5 & 367.4 & 385.9 \\
\cline { 2 - 8 } & Finite element & 92.5 & 200.7 & 263.6 & 365.4 & 381.6 \\
\cline { 2 - 7 } & Error (\%) & 1.5 & 1.4 & 0.4 & 0.5 & 1.1 \\
\hline \multirow{3}{*}{2.0} & Present method & 171.3 & 273.7 & 447.1 & 575.3 & 671.9 \\
\cline { 2 - 7 } & Finite element & 181.1 & 284.8 & 461.6 & 612.4 & 707.3 \\
\cline { 2 - 7 } & Error (\%) & 5.4 & 3.9 & 3.1 & 6.1 & 5.0 \\
\hline
\end{tabular}




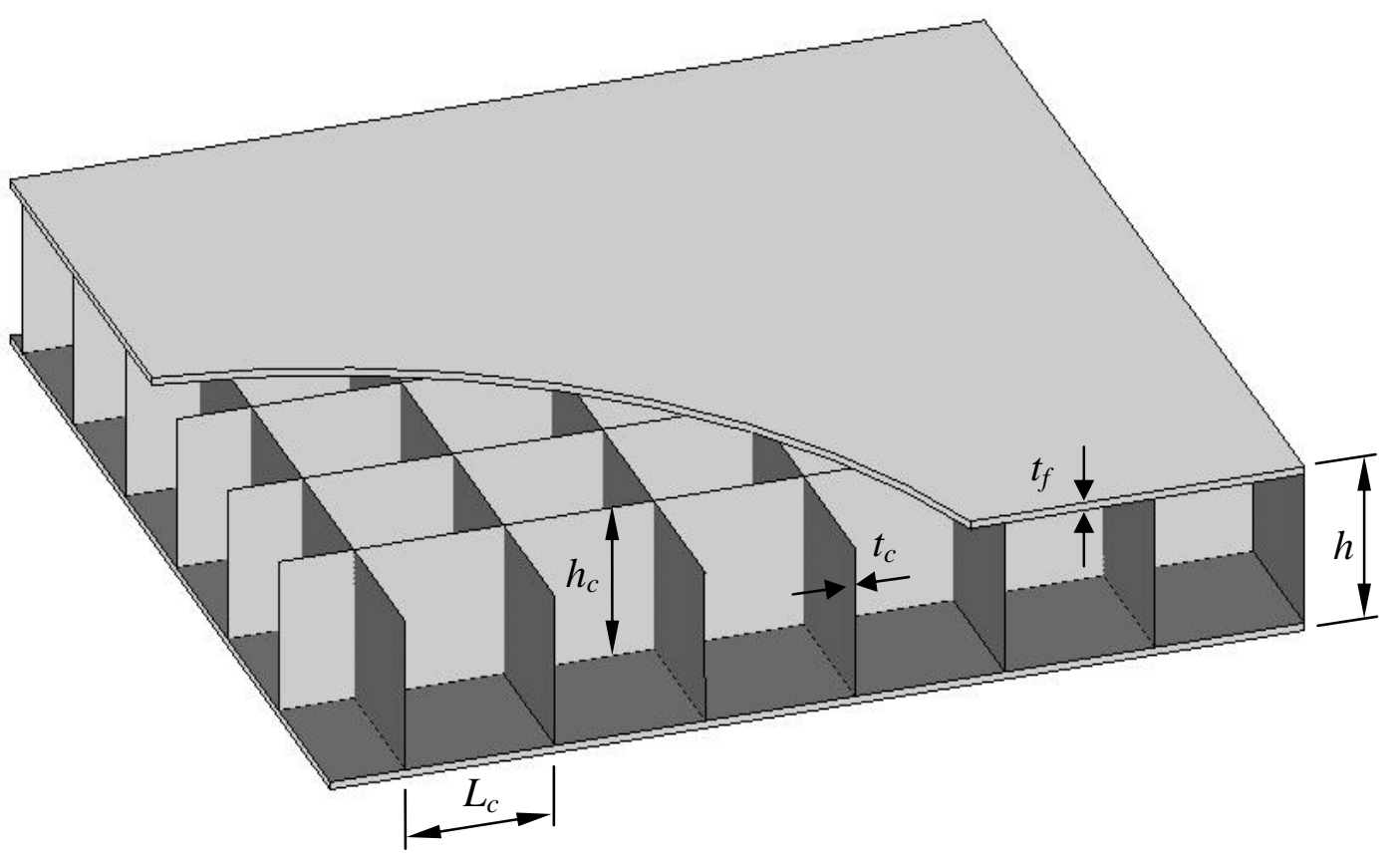

Figure 1. Key dimensions of a square honeycomb panel.

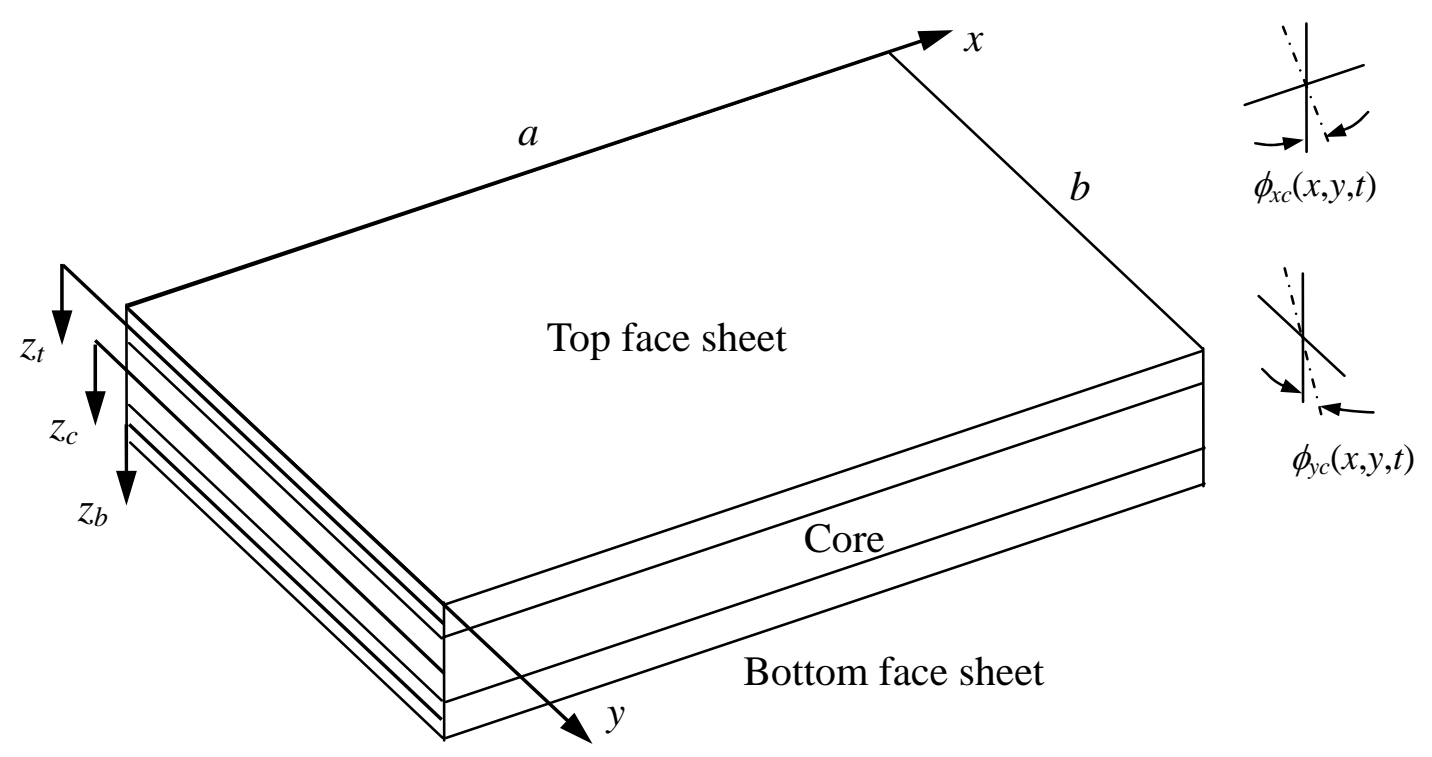

Figure 2. Coordinate system for square honeycomb panel 


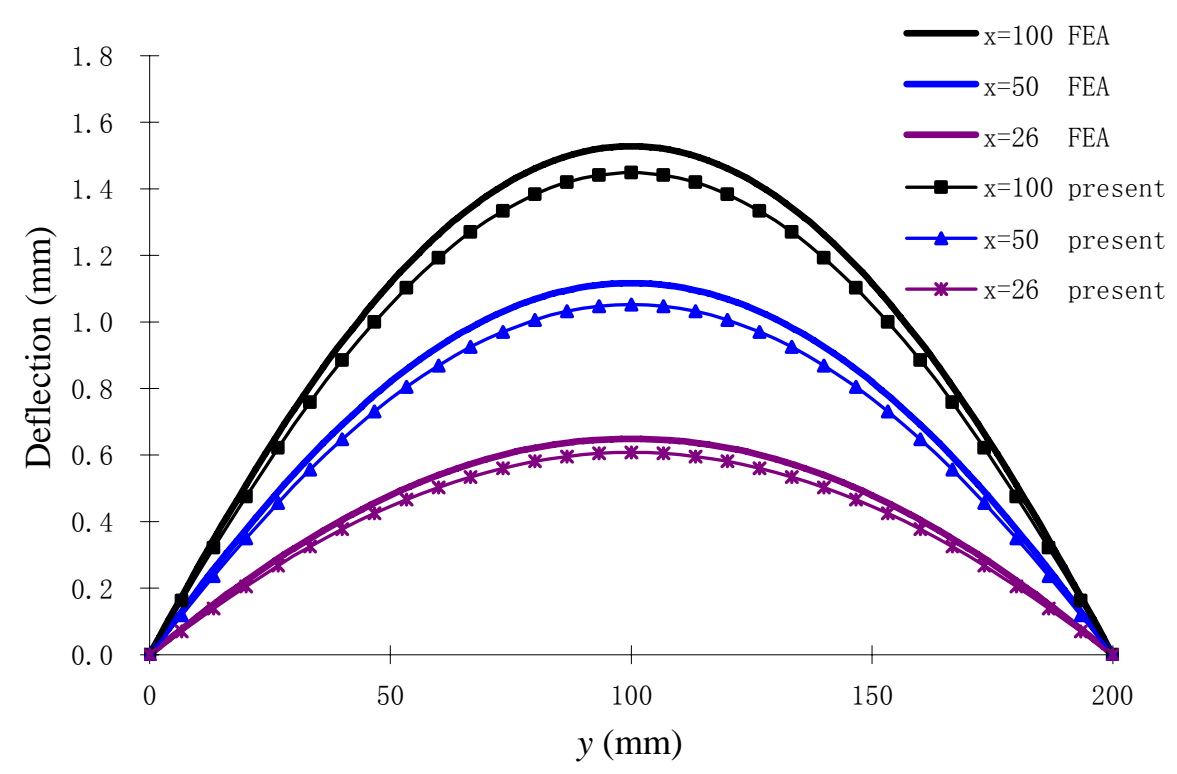

Figure 3. Deflection at $x=100 \mathrm{~mm}, 50 \mathrm{~mm}, 26 \mathrm{~mm}$ for $\rho_{r}=20 \%$

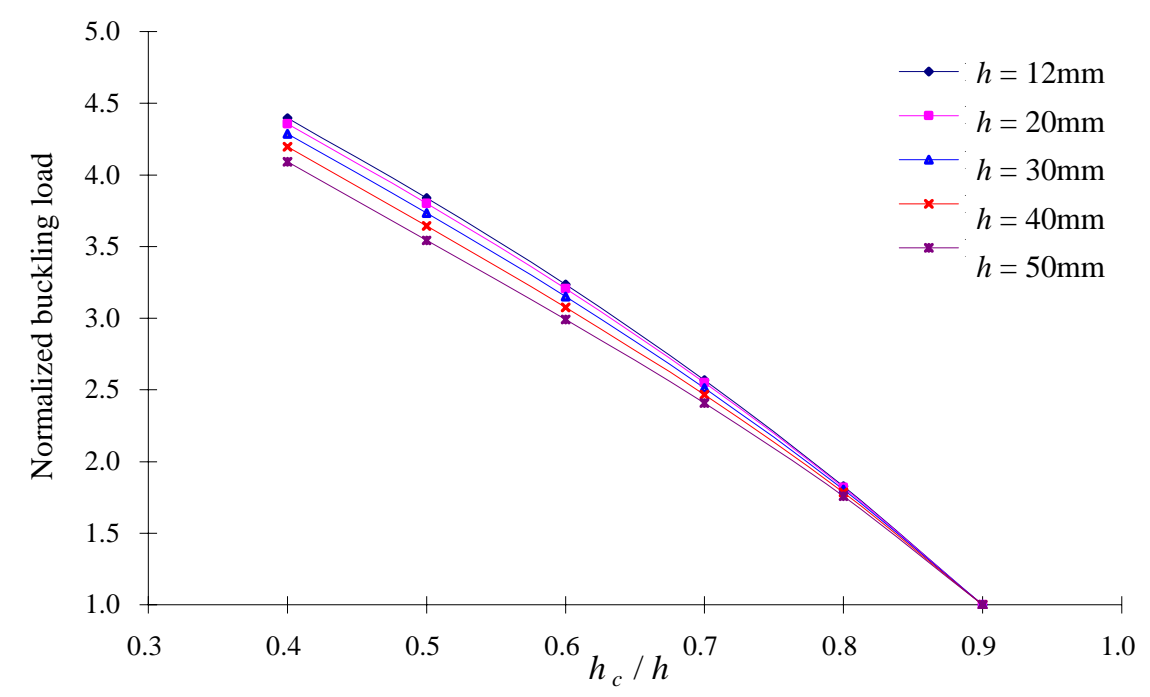

Figure 4. Effect of core height on buckling load of sandwich panels with square-honeycomb core 


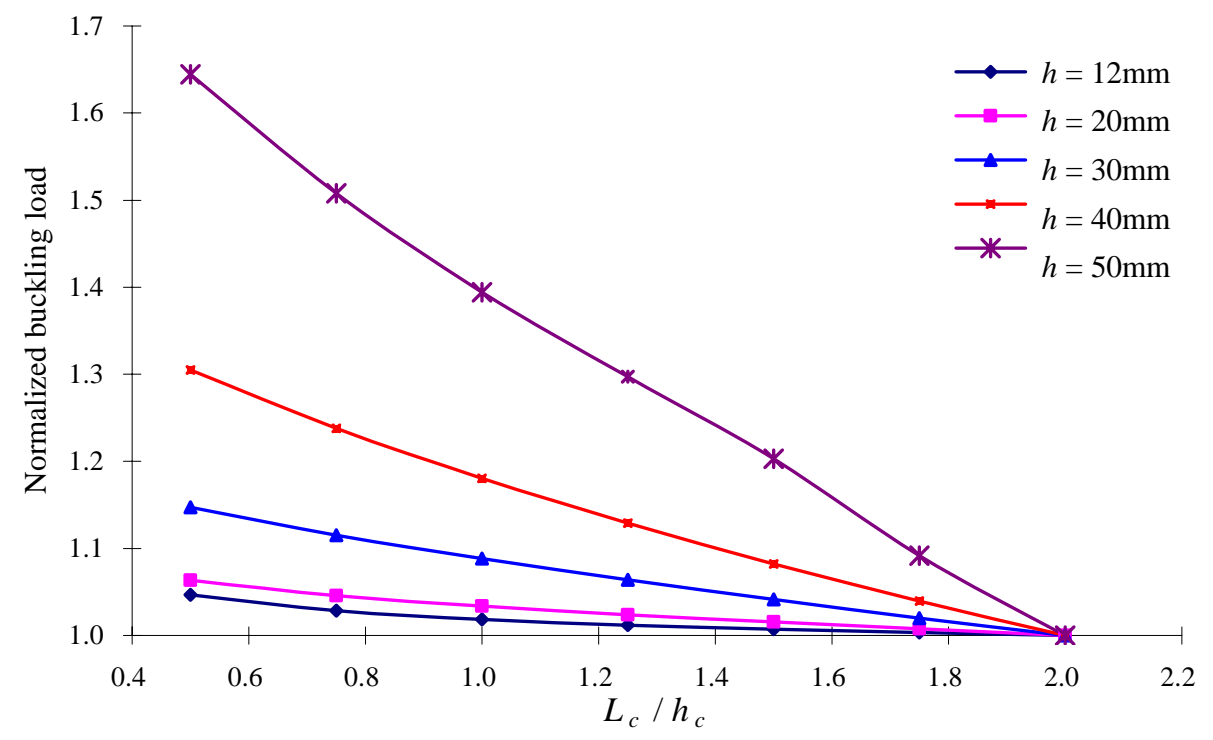

Figure 5. Effect of core sheet spacing on buckling load of sandwich panels with square-honeycomb core

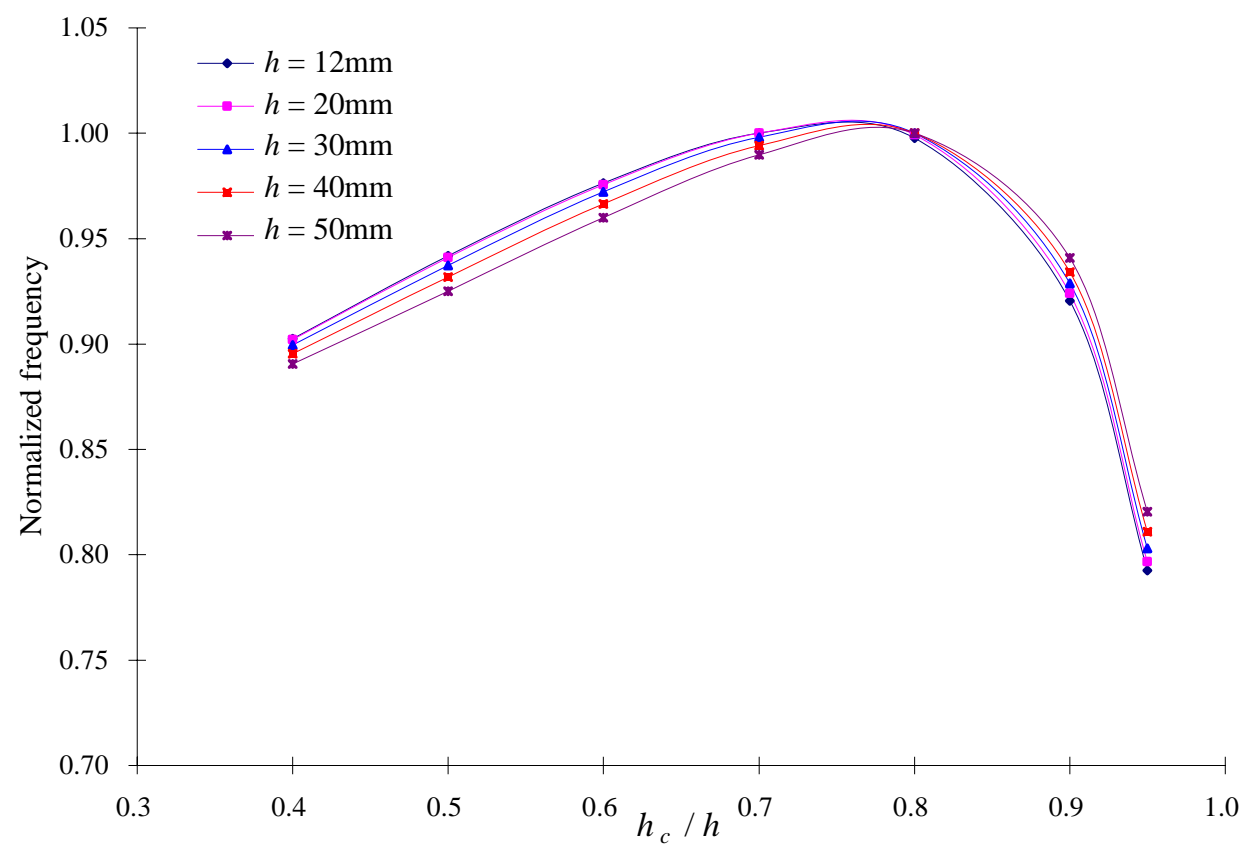

Figure 6. Effect of core height on the first natural frequency of sandwich panels with square-honeycomb core 


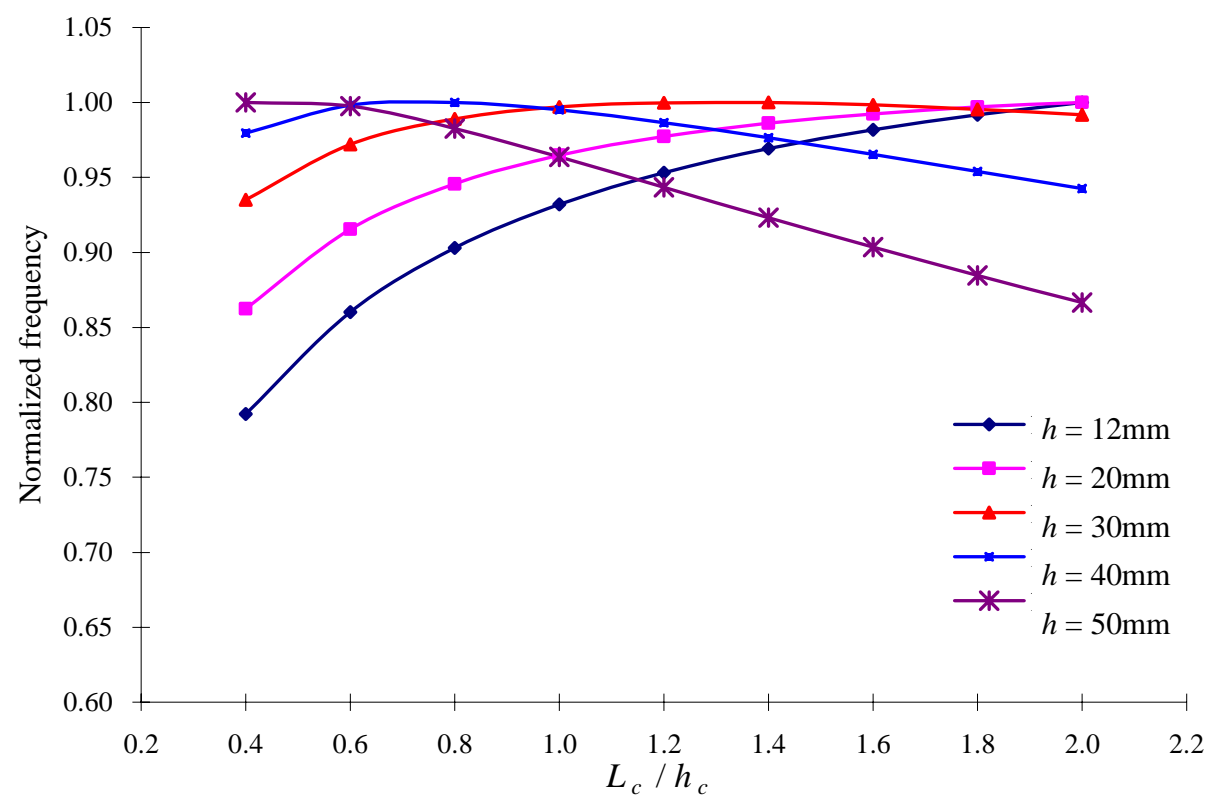

Figure 7. Effect of core sheet spacing on the first natural frequency of sandwich panels with square-honeycomb core of $h_{c} / h=0.8$ 